\title{
Unilateral Left Pulmonary Edema Caused by Contained Rupture of the Ascending Aortic Dissection
}

\author{
Kazuhito Hirata ${ }^{1}$, Toru Ishimine ${ }^{2}$, Izumi Nakayama ${ }^{3}$, Nobuhito Yagi ${ }^{1}$, Minoru Wake ${ }^{1}$, \\ Takanori Takahashi ${ }^{1}$, Naoki Taniguchi ${ }^{2}$ and Toshiho Tengan ${ }^{2}$
}

\begin{abstract}
:
Unilateral pulmonary edema (UPE) is a rare entity and is usually associated with severe mitral regurgitation and more frequently occurs in the right lung. We herein report a case of unilateral left pulmonary edema caused by external compression of the right pulmonary artery and left pulmonary vein caused by the presence of hematoma due to type A acute aortic dissection (AAD), resulting in asymmetrically increased inflow and decreased outflow in the left pulmonary circulation. Physicians caring for patients with UPE should be aware that AAD leading to the external compression of the heart may be a possible underlying mechanism.
\end{abstract}

Key words: unilateral pulmonary edema, acute aortic dissection, heart failure

(Intern Med 60: 751-753, 2021)

(DOI: 10.2169/internalmedicine.5750-20)

\section{Introduction}

Unilateral pulmonary edema (UPE) is a rare entity usually associated with severe mitral regurgitation and more frequently occurs in the right lung than in the left $(1,2)$. We herein report a case of unilateral left pulmonary edema caused by external compression of the right pulmonary artery and left pulmonary vein caused by the presence of a hematoma due to type A acute aortic dissection (AAD).

\section{Case Report}

A man in his mid-60s was brought to our emergency room with chest pain and dyspnea persistent for 1 day.

His medical history was remarkable for aortic valve replacement (Björk-Shiley single tilting disc valve) due to aortic valve stenosis and regurgitation complicated with infective endocarditis and cerebral infarction in his late 20s in 1979. Since then, he had suffered two more episodes of cerebrovascular events, namely a right brain infarction in 1988 and left cerebellar hemorrhage in 2012. As a result, his daily activities were very limited, and he was currently living in a nursing home. He was oriented and could commu- nicate, but he could not walk and thus used a wheelchair. $\mathrm{He}$ had been treated with enalapril $5 \mathrm{mg}$ daily, bisoprolol $2.5 \mathrm{mg}$ daily, and warfarin $6.5 \mathrm{mg}$ daily. His normal blood pressure (BP) was 120-130/70 mmHg.

$\mathrm{He}$ had been in his usual state of health until 17:00 on the day before admission, when he had suffered an episode of chest pain and dyspnea for 5 minutes. At 15:00 on the day of admission, he again developed chest pain associated with cold sweating. His BP was $80 / 50 \mathrm{mmHg}$, and his heart rate (HR) was 80 beats $/ \mathrm{min}$. He was taken to our emergency room by ambulance.

His initial vital signs were as follows: BP $122 / 70 \mathrm{mmHg}$, HR 98 beats/min, respiratory rate 20/min, and body temperature $37.1{ }^{\circ} \mathrm{C}$. Chest X-ray showed cardiomegaly, mediastinal widening, and bilateral pulmonary venous congestion (Fig. 1A). A 12-lead electrocardiogram revealed sinus tachycardia with a HR of 100 beats/min, left bundle branch block, and occasional premature atrial contractions. The $\mathrm{T}$ wave was inverted in leads I, II, aVL, aVF, and V4-V6, but no ST elevation suggestive of myocardial infarction was noted. Bedside echocardiography revealed a preserved left ventricular systolic function, mild perivalvular aortic regurgitation, mild mitral regurgitation and dilated aortic root. A localized left ventricular apical aneurysm was noted, but there

${ }^{1}$ Division of Cardiology, Okinawa Chubu Hospital, Japan, ${ }^{2}$ Cardiovascular Surgery, Okinawa Chubu Hospital, Japan and ${ }^{3}$ Intensive Care Unit, Okinawa Chubu Hospital, Japan

Received: July 1, 2020; Accepted: August 25, 2020; Advance Publication by J-STAGE: October 7, 2020

Correspondence to Dr. Kazuhito Hirata, kheart911@yahoo.co.jp 


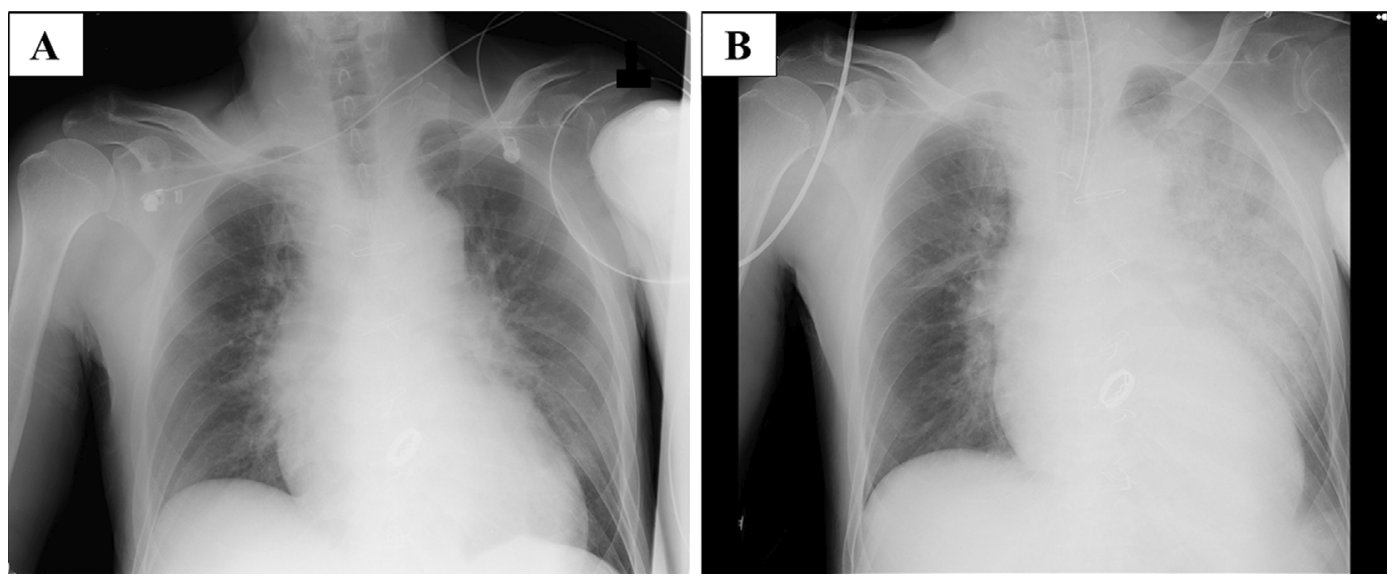

Figure 1. A: Initial chest X-ray showing cardiomegaly, mediastinal widening, and bilateral pulmonary venous congestion. B: Chest $X$-ray 30 hours after hospitalization shows unilateral left pulmonary edema. The patient developed hemodynamic collapse and was intubated.


Figure 2. A: Initial contrast-enhanced CT scan showing a dilated aortic root and an intimal flap. A contained rupture and mediastinal hematoma compress the upper pulmonary veins and upper roof of the left atrium. B, C: Blood flow to the right and left pulmonary arteries is maintained. Ao: Ascending aorta, RPV: Right pulmonary vein, LPV: left pulmonary vein, H: Hematoma, LA: Left atrium, RPA: Right pulmonary artery, LPA: Left pulmonary artery

was no pericardial effusion.

To evaluate possible aortic dissection and apical aneurysm, contrast-enhanced computed tomography (CT) was performed. The aortic root was dilated, and there was a classic intimal flap in the ascending aorta (Fig. 2A). Notably, there was a contained rupture to the posterior mediastinal space. The apical aneurysm was small and very localized and was attributed to the chronic "vent aneurysm" from the previous aortic valve replacement (an apical vent was commonly performed in the 1970s). The upper part of the left atrium and bilateral upper pulmonary vein was compressed by the dilated ascending aorta and the contained hematoma (Fig. 2B, C). Because surgical treatment was considered to carry a high risk of mortality due to his medical and social history, the heart team decided to manage him medically.

At midnight on hospital day 2 (30 hours after admission), he suddenly developed severe dyspnea and cyanosis with shock. His BP fell to $75 / 50 \mathrm{mmHg}$, and his HR increased to $130 \mathrm{bpm}$ with new-onset atrial fibrillation. There was no ST elevation. Chest X-ray revealed unilateral left pulmonary edema (Fig. 1B). He was intubated, and noradrenaline was used with fluid expansion.

Repeated contrast-enhanced CT revealed an increased size of the hematoma, which was now severely compressing the right pulmonary artery and left pulmonary vein (Fig. 3B, C). Emergency surgical ascending aortic replacement and removal of the hematoma were performed. The UPE improved immediately after the surgery, and the postoperative course was uneventful. The patient was discharged to a nursing home on day 27.

\section{Discussion}

UPE is a rare entity $(2.1 \%$ of cardiogenic pulmonary edema) and is frequently misdiagnosed as pneumonia, leading to a delay in initiating heart failure therapy and unnecessary antibiotic treatment $(1,2)$. UPE is usually associated with severe mitral regurgitation and more frequently occurs in the right lung than in the left $(1,2)$. The eccentric direction of the mitral regurgitant jet may impede local pulmo- 

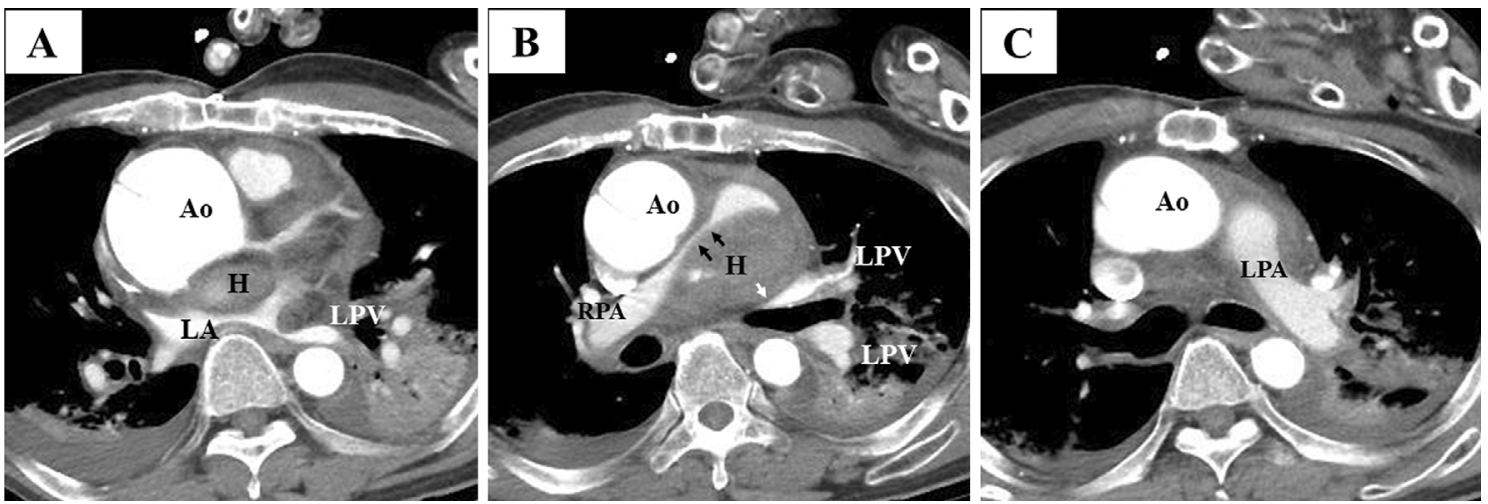

Figure 3. A: The mediastinal hematoma increased in size, further compressing the upper roof of the left atrium. B, C: The right pulmonary artery (black arrows) and left pulmonary vein (a white arrow) are severely narrowed by external compression of the hematoma, while the left pulmonary artery is intact. Ao: Ascending aorta, RPV: Right pulmonary vein, LPV: Left pulmonary vein, H: Hematoma, LA: Left atrium, RPA: Right pulmonary artery, LPA: Left pulmonary artery

nary venous flow, resulting in unilateral elevation of the pulmonary venous pressure $(1,2)$. A longer right-side lymphatic tract into the thoracic duct for drainage may also play an important role in the development of right-side UPE (1).

Other causes of UPE include acute pulmonary emboli, reexpansion pulmonary edema, and external compression of the pulmonary vein or artery by a mediastinal tumor, resulting in an asymmetric inflow to the pulmonary arteries or outflow in the pulmonary vein (3-5). Acute aortic dissection causes compression of the left atrium, pulmonary artery, or pulmonary vein, leading to symptoms and signs mimicking heart failure, pulmonary emboli, and pulmonary stenosis (6-11). However, UPE caused by acute aortic dissection is extremely rare $(12,13)$. In the present case, the bilateral upper pulmonary veins and upper left atrium were initially compressed between the dilated ascending aorta and the contained hematoma, resulting in bilateral pulmonary venous congestion (Fig. 1A). As the size of the hematoma increased, unilateral left pulmonary edema was caused by the simultaneous compression of the right pulmonary artery and left pulmonary vein, resulting in an asymmetrically increased inflow and decreased outflow in the left pulmonary circulation (Fig. 1B). Surgical management was lifesaving.

\section{Conclusion}

Physicians taking care of patients with UPE should be aware that acute aortic dissection leading to the external compression of the structure of the heart may be a possible underlying mechanism of UPE.

The authors state that they have no Conflict of Interest (COI).

\section{References}

1. Attias D, Mansencal N, Auvert B, et al. Prevalence, characteris- tics, and outcomes of patients presenting with cardiogenic unilateral pulmonary edema. Circulation 122: 1109-1115, 2010.

2. Inotani S, Kubokawa S, Nakaoka Y, et al. Unilateral cardiogenic pulmonary edema. J Cardiol Cases 17: 1785-1788, 2018.

3. Koroscil M. Tumbling downhill: unilateral pulmonary edema. Am J Med 129: 1054-1056, 2016.

4. Sohara Y. Reexpansion pulmonary edema. Ann Thorc Cardiovasc Surg 14: 205-209, 2008.

5. Marshall ME, Trump DL. Acquired extrinsic pulmonary stenosis caused by mediastinal tumors. Cancer 49: 1496-1499, 1982.

6. Walpot J, Amsel B, Pasteuning WH, Olree M. Left atrial compression by dissecting aneurysm of the ascending aorta. J Am Soc Echocardiogr 20: 1220. e4-1220. e6, 2007.

7. Bonser RS, Abudhaise H, Bashir M, Ranasingheet AM. Left atrial compression secondary to contained rupture of type A aortic dissection. Circulation 123: e592-e593, 2011.

8. Bhatt K, Navia J, Flamm S, Bolen M. Type A aortic dissection mimicking a saddle pulmonary embolus on computed tomographic angiography. Circulation 129: 2180-2182, 2014.

9. Masuo M, Takano H, Takamoto S, Tanaka J, Kitamura S, Saito T. Pulmonary artery obstruction caused by thoracic aortic dissection. A case with unique pathological findings. Circ J 68: 392-395, 2004.

10. De Silva RJ, Hosseinpour R, Screaton N, Stoica S, Goodwin AT. Right pulmonary artery occlusion by an acute dissecting aneurysm of the ascending aorta. J Cardiothorac Surg 1: 29-32, 2006.

11. Yamazaki S, Funakoshi H. Pulomonary artery stenosis due to aortic dissection. Eur Heart J Cardiovasc Imaging 20: 1019, 2019.

12. Kagele SF, Charan NB. Unilateral pulmonary edema: an unusual cause. Chest 102: 1279-1280, 1992.

13. Takahashi M, Ikeda U, Shimada K, Takeda H. Unilateral pulmonary edema related to pulmonary artery compression resulting from acute dissecting aortic aneurysm. Am Heart J 126: 1225$1227,1193$.

The Internal Medicine is an Open Access journal distributed under the Creative Commons Attribution-NonCommercial-NoDerivatives 4.0 International License. To view the details of this license, please visit (https://creativecommons.org/licenses/ by-nc-nd/4.0/).

(C) 2021 The Japanese Society of Internal Medicine Intern Med 60: 751-753, 2021 